\title{
Impacts of injection temperature on the relevant heat transport processes in groundwater heat pump (GWHP) systems
}

\author{
Byeong-Hak Park
}

\author{
Kang-Kun Lee*
}

\begin{abstract}
In many hydrogeological applications, the influence of temperature on fluid density and viscosity have often been neglected. However, high contrasts in temperature which occurs in the field applications such as groundwater heat pump (GWHP) systems, can make the effects of variable density and viscosity on flow and transport significant. A theoretical study suggests that free convection occurs in an infinitely extensive horizontal layer when the Rayleigh number exceeds about 40. Experimental investigations are still lacking on the conditions where the influence of temperature can be important.

In this study, a laboratory experimental system was developed to investigate the impacts of injection temperature on the relevant heat transport processes. First of all, the experiments such as sieve analysis and constant-head permeability test were performed to estimate the physical properties of the saturated porous medium. Laboratory tracer tests using a resistor as a beat source were conducted with/ without background flow conditions to derive the thermal properties of the medium. Lastly, tracer tests using injected water with different temperatures were performed to identify the certain conditions where the variations in fluid density and viscosity play an important role in the subsurface flow and transport.
\end{abstract}

\section{INTRODUCTION}

In many hydrogeological applications, the temperature dependency of fluid properties is often assumed to be negligible. However, high temperature difference which occurs in the field applications such as groundwater heat pump (GWHP) systems, can make the effects of variable density and viscosity on flow and transport significant. A theoretical study suggests that free convection occurs in an infinitely extensive horizontal layer when the Rayleigh number exceeds about 40 (Domenico and Schwartz, 1998). Experimental investigations are still lacking on the threshold conditions where the influence of temperature should be considered.

In this study, a laboratory experimental system which can simulate the subsurface flow and heat transport was designed to examine the impacts of injection temperature on thermal plume propagation. First of all, preexperiments were performed to estimate the physical properties of the saturated porous medium. Heat tracer tests using a resistor were conducted at various background flow velocities to derive the thermal properties of the medium. Lastly, heat tracer tests using injected water with different temperatures were performed to identify the certain conditions where the variations in fluid density and viscosity play an important role in the subsurface flow and transport.

\section{Methodology}

Experimental design. The experimental system mainly consists of the acrylic glass tank filled with fullysaturated sand. The size of the sand tank is $1.3 \mathrm{~m} \times 0.6 \mathrm{~m} \times 0.8 \mathrm{~m}(\mathrm{~L} \times \mathrm{W} \times \mathrm{H})$ and the tank is devided into three 
chambers: two constant-head chambers on both sides and one chamber in the middle filled with sand (see Fig. 1). By changing the water level at constant-head chambers, background flow rate was adjusted in the experiments. Figure 1b shows the location of heat sources and temperature sensors installed in the middle chamber. Two different heat sources of a resistor and injected water were used as a heat tracer. In a case of heat tracer tests using injected water, hot water in a thermostatic barrel was injected by a peristaltic pump into an injection well inside the middle chamber. Temperature change was detected by RTD sensors and logged in a data aquisition system developed in the LabVIEW 2009 programming environment.
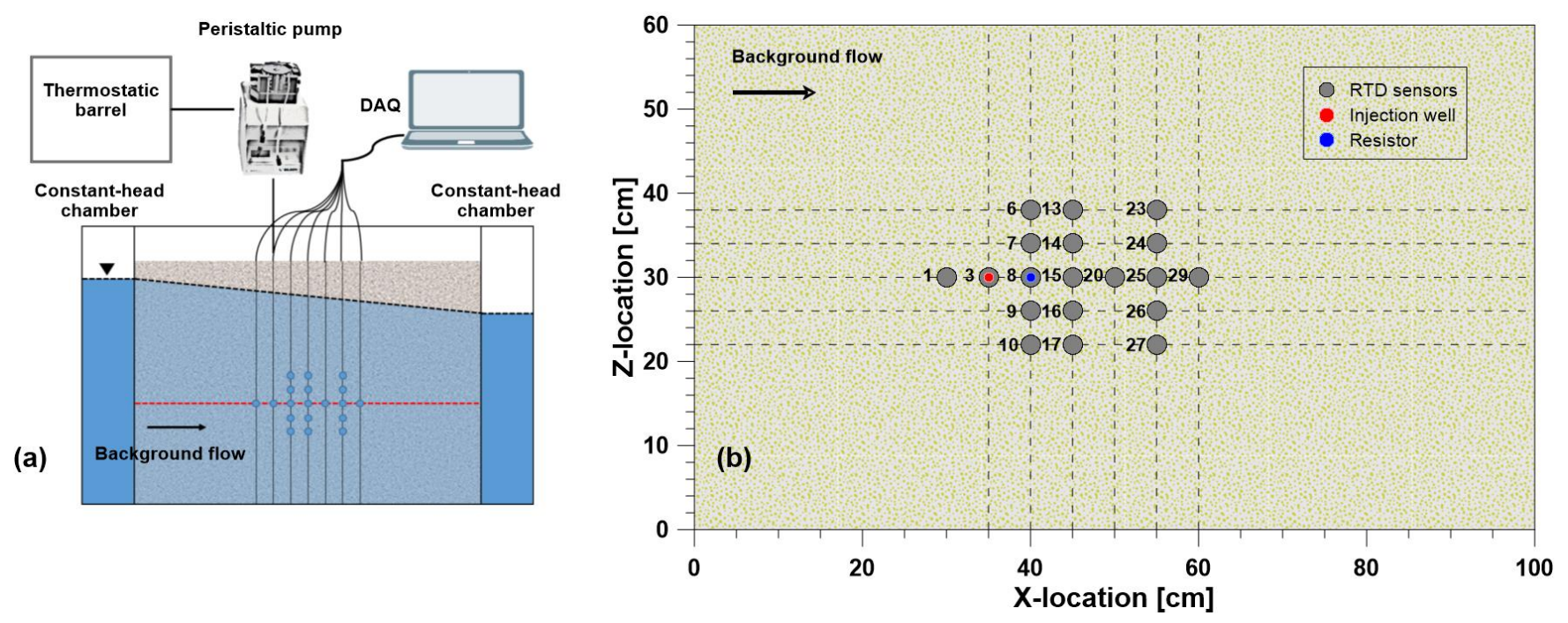

Figure 1 (a) Schematic diagram of a laboratory experimental system and (b) location of heat sources and RTD sensors.

Properties of porous medium. Before heat tracer tests were conducted, preexperiments were performed to estimate the physical properties of sand. Sieve analysis were performed to evaluate the mean grain size and uniformity of the porous medium. The analysis results showed that the mean diameter of the sand is $0.52 \mathrm{~mm}$ and its uniformity is 1.41 , indicating relatively homogeneous material. The porosity of the sand was determined to be about 0.3306 by measuring the moisture contents in the experimental setup. Static permeability tests were repeatedly conducted with different hydraulic gradients. The linear regression method was applied to the measured hydraulic gradients and specific discharges, and hydraulic conductivity of the sand was estimated to be $2.041 \times 10^{3} \mathrm{~m} / \mathrm{s}$ with high $\mathrm{R}^{2}$ value over 0.9981 . The estimated properties of the sand are listed in Table 1.

Table 1. Hydrogeologic and thermal properties of sand

\begin{tabular}{cccc}
\hline Properties & Unit & Value $(\mathbf{m} \pm \boldsymbol{\sigma})$ & Source/comment \\
\hline \hline Mean grain size $\left(\mathrm{d}_{50}\right)$ & $\mathrm{mm}$ & $0.52 \pm 0.01$ & Sieve analysis \\
Uniformity $(\mathrm{U})$ & - & $1.41 \pm 0.01$ & Sieve analysis \\
Porosity $(\mathrm{n})$ & - & 0.3306 & Moisture content \\
Hydraulic conductivity $(\mathrm{K})$ & $10^{-3} \mathrm{~m} / \mathrm{s}$ & 2.041 & Permeability test \\
Thermal conductivity of sand & $\mathrm{W} / \mathrm{m}-\mathrm{K}$ & $3.383 \pm 0.204$ & Resistor test \\
Vol. heat capacity of sand & $10^{6} \mathrm{~J} / \mathrm{m}^{3}-\mathrm{K}$ & $2.078 \pm 0.081$ & Resistor test
\end{tabular}


Laboratory heat tracer test. In this study, two different heat sources of a resistor and injected water were used as a thermal tracer to investigate the effect of injection temperature on thermal plume propagration in the subsurface. First, heat tracer tests using a resistor were performed with a constant heat source of $4.517 \mathrm{~W}$ without background flow to estimate the thermal conductivity and volumetric heat capacity of the porous medium. Then, heat tracer tests using a resistor were conducted at various background flow rates ranging from $5.301 \times 10^{-5}$ to $1.448 \times 10^{-4} \mathrm{~m} / \mathrm{s}$. Lastly, hot water $5-15 \mathrm{~K}$ higher than background water was injected into the porous medium with an injection rate of about $100 \mathrm{ml} / \mathrm{min}$.

The observed temperature breakthrough curves were analyzed by a curve-fitting procedure with analytical models. The resistor tests under no-flow condition were analyzed by a continuous point source (CPS) model expressed in Equation 1 (Carslaw and Jaeger, 1959; Stauffer et al., 2013) and thermal properties of the sand were evaluated. Equation 2, called a moving continous point source (MCPS) model, was used to interpret the resistor tests with various background flow rates (Carslaw and Jaeger, 1959; Rau et al., 2012), and thermal dispersion coefficients and thermal front velocities were derived from the analysis results. Equation 2 was also applied to the thermal breakthrough curves obtaind from injection tests. In the analysis, the quality of fit was assessed by the root mean square error (RMSE) value (Equation 3), and thermal properties were determined from the best-fitted model minimizing the RMSE value. More details on the parameter estimation procedure can be found in the recent literature (Park et al., 2018). To quantify a transport regime, the thermal peclet number, characterizing the relative contribution of convective and conductive heat transport, was defined as in Equation 4 (de Marsily, 1986; Anderson, 2005; Rau et al., 2014).

$$
\begin{gathered}
T(x, y, z, t)=T_{0}+\frac{Q}{4 \pi \lambda R^{\prime}} \operatorname{erfc}\left(\frac{R^{\prime}}{\sqrt{4 D^{t} t}}\right) \\
T(x, y, z, t)=T_{0}+\frac{Q}{8 \pi D_{T}^{t} \rho c R} \exp \left(\frac{x V^{t}}{2 D_{L}^{t}}\right) \cdot\left[\exp \left(-\frac{R v^{t}}{2 D_{L}^{t}}\right) \operatorname{erf}\left(\frac{R-v^{t} t}{2 \sqrt{D_{L}^{t} t}}\right)+\exp \left(\frac{R v^{t}}{2 D_{L}^{t}}\right) \operatorname{erf}\left(\frac{R+v^{t} t}{2 \sqrt{D_{L}^{t} t}}\right)\right] \\
R M S E=\sqrt{\sum_{i=1}^{n} \frac{\left(T_{o, i}-T_{m, i}\right)^{2}}{n}} \\
P e^{t}=\frac{\rho_{w} C_{w} q d_{50}}{\lambda}
\end{gathered}
$$

\section{RESULTS}

Figure 2 shows the thermal breakthrough curves observed at center and off-center locations (cross symbol) and the CPS model results (solid lines). As can be seen in Figure 2, the best-fitted model agrees very well with the oberved temperature data (RMSE $<0.007 \mathrm{~K}$ ). From the best fit between the observations and modeling results, the thermal conductivity and volumetric heat capacity of sand were estimated to be $3.185-3.878 \mathrm{~W} / \mathrm{m}-\mathrm{K}$ and $1.770-2.190 \mathrm{MJ} / \mathrm{m}^{3}$ $\mathrm{K}$, respectively. Based on the estimated thermal properties, resistor tests performed at various background flow rates were analyzed with the MCPS model. 


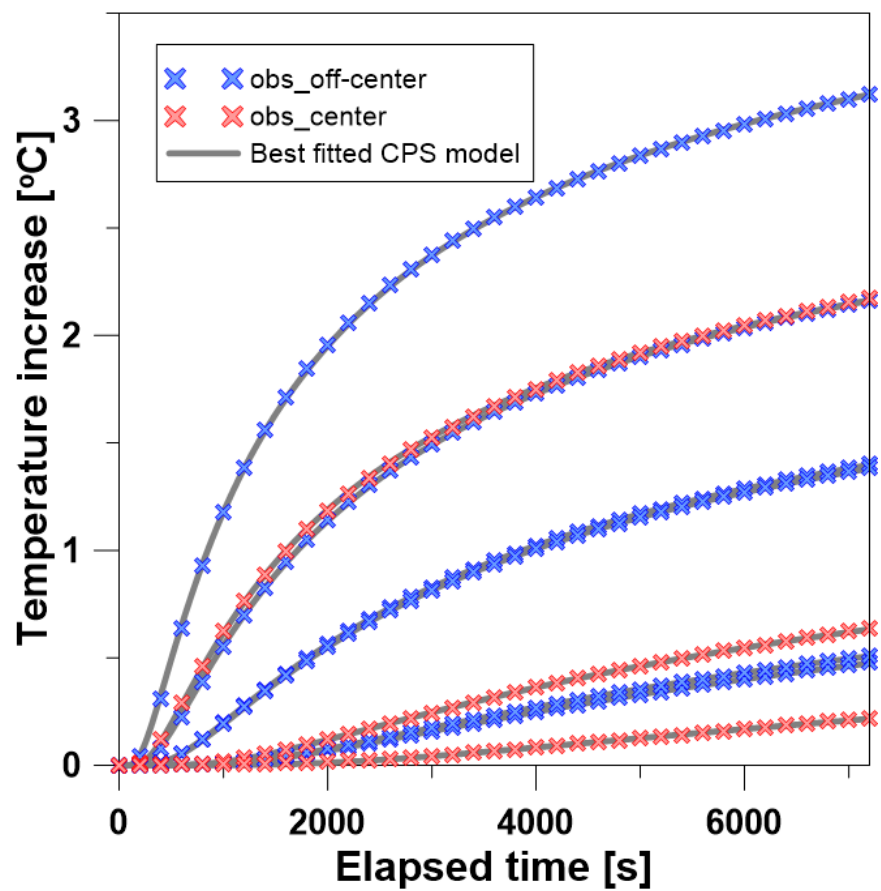

Figure 2 Thermal breakthrough curves observed at center and off-center locations (cross symbol) and best-fitted CPS models.
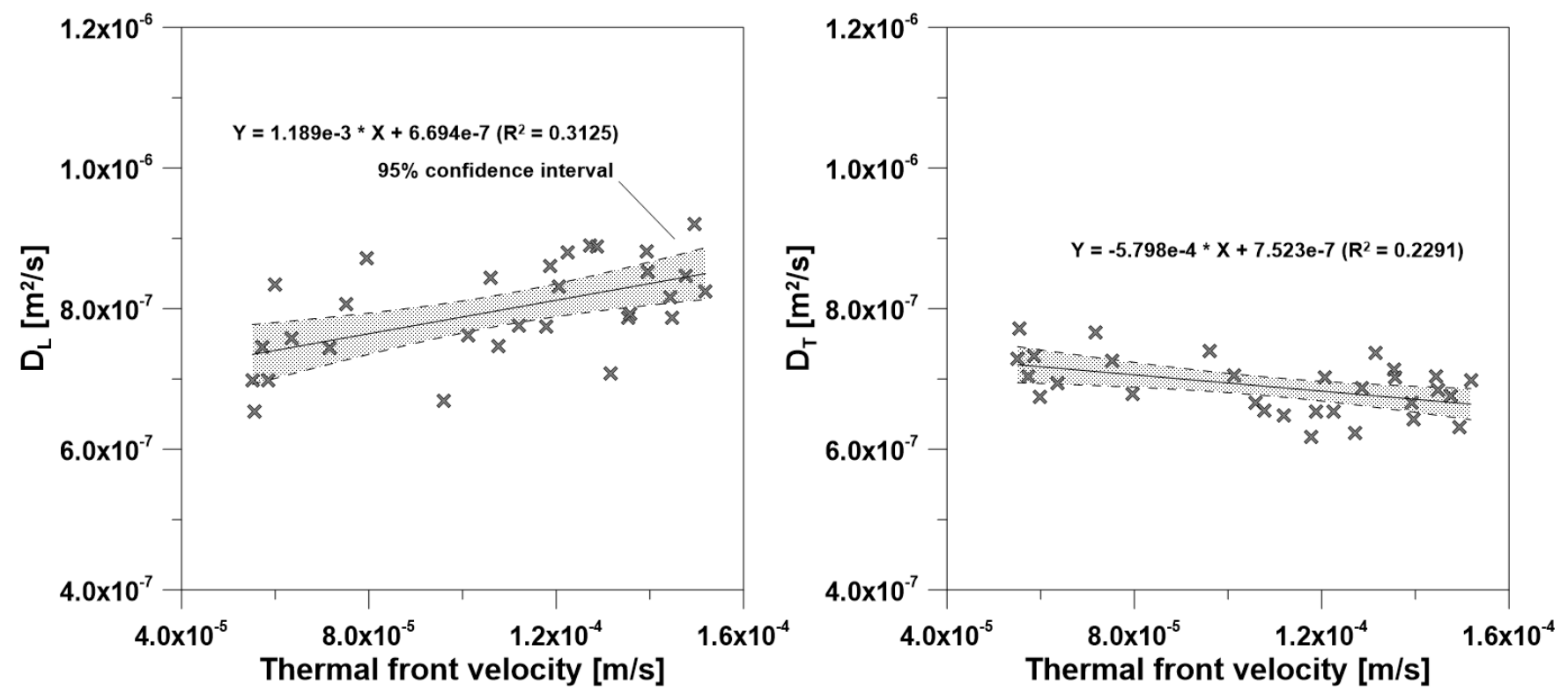

Figure 3 Relationships between the thermal dispersion coefficients and thermal front velocities: (a) longitudinal and (b) transverse directions. 
Figure 3 describes the relationships between the thermal dispersion coefficients and thermal front velocities derived from the best-fitted MCPS model. The results showed that the longitudinal thermal dispersion coefficients increase with flow velocity, while the transverse thermal dispersion coefficients decrease with flow velocity. However, there were a lot of scatter in the relations with low $\mathrm{R}^{2}$ values of 0.3125 (longitudinal) and 0.2291 (transverse). Such scatter can be partly caused by low flow velocities as explained in previous studies (Yuan et al., 1991; Rau et al., 2012). Although we tried to increase hydraulic gradient, it was found that thermal peclet numbers (Pet) for all the tests are less than 0.2. In the conduction dominant regime $\left(\mathrm{Pe}^{t}<1\right)$, the effective thermal diffusivity is the dominant part of the thermal dispersion coefficient, and the contribution of flow velocity to that is very small (Yuan et al., 1991).

Injection tests were also analyzed by the MCPS model in Equation 2. Figure 4 describes the thermal front velocities estimated from the resistor (black cross) and injection (colored symbol) experiments at various background flow velocities. As shown in Figure 4, the estimated thermal front velocities from hot injection tests were higher than those from resistor tests. There can be two reasons for that. Even when the temperature difference is small $(\Delta \mathrm{T}=5$ $\mathrm{K})$, the flow velocity was estimated to be faster. This indicates that injection increases the background flow velocity. When focusing on the injection tests results (colored symbols in Figure 4), themal front velocities have a higher value with increaing temperature. This is because hydraulic conductivity, which is a function of fluid density and viscosity, increases with temperature.

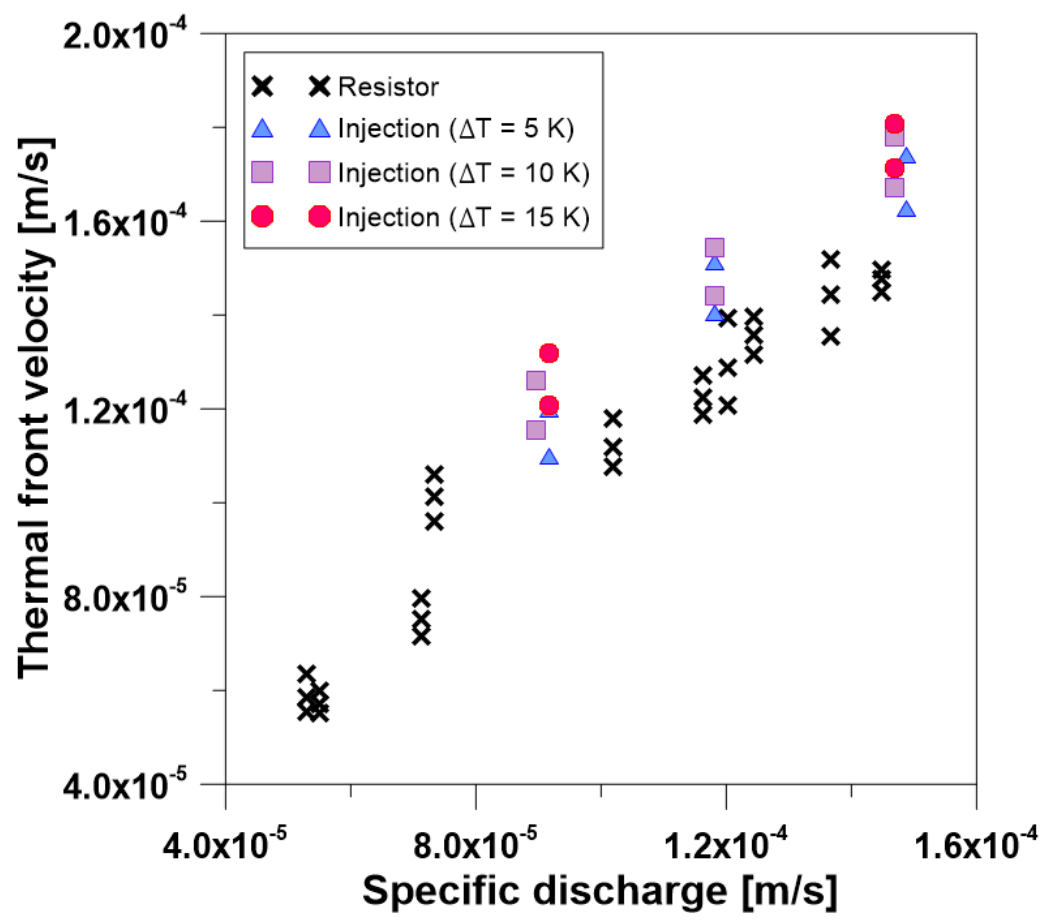

Figure 4 Thermal front velocities estimated from the resistor (black cross) and injection (colored symbol) experiments at various background flow velocities. 


\section{CONCLUSION}

In this study, the laboratory experimental system was developed to investigate the effect of the temperature dependency of fluid properties on the relevant heat transport processes in GWHP systems. First, preexperiments were conducted to estimate the physical properties of the porous medium. Then, heat tracer tests using two different heat sources of a resistor and hot water were performed to estimate the thermal properties of the sand and to examine the influence of injected hot water on thermal plume propagation. The observed thermal breakthrough curves were repeatedly analyzed by the analytical (CPS and MCPS) models in Equation 1 and 2. The analysis results of resistor tests showed that the thermal dispersion coefficient has an unclear tendency with low $\mathrm{R}^{2}$ values $(<0.32)$ and a lot of scatter. This is because heat tracer tests in this study was conducted in conduction dominated regime $\left(\operatorname{Pe}^{t}<0.2\right)$ despite the effort to increase the hydraulic gradient. The thermal front velocities analyzed from injection tests suggest the effect of the injection and temperature difference on the thermal plume propagation. Further experimental and numerical study will be performed to investigate the vertical impacts as well as horizontal effects of variable fluid properties.

\section{ACKNOWLEDGMENTS}

This work was supported by the National Research Foundation of Korea (NRF) grant funded by the Korea government (MSIP) (No. 2017R1A2B3002119).

\section{NOMENCLATURE}

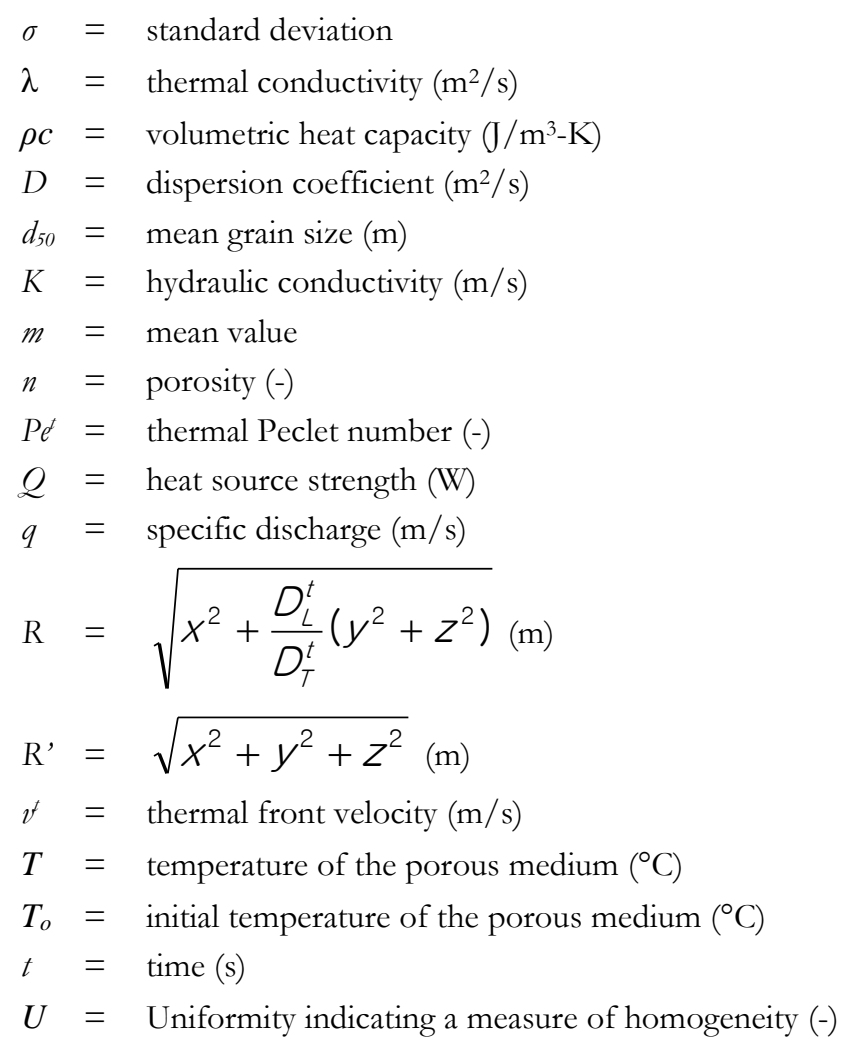

\section{Supscripts}

$t=$ thermal 


\section{Subscripts}

$$
\begin{aligned}
L & =\text { longitudinal } \\
T & =\text { transverse } \\
m & =\text { modeling result } \\
o & =\text { observation } \\
s & =\text { solid property } \\
w & =\text { water property }
\end{aligned}
$$

\section{REFERENCES}

Carslaw, H.S., and Jaeger, J.C. 1959. Conduction of heat in solids. NY: Oxford University Press.

Domenico, P. A., and Schwartz, F. W. 1998. Physical and chemical hydrogeology. New York: Wiley.

Park, B. H., Lee, B. H., and Lee, K. K. 2018. Experimental investigation of the thermal dispersion coefficient under forced groundwater flow for designing an optimal groundwater beat pump (GWHP) system. Journal of Hydrology. 562: 385-396.

Rau, G.C., Andersen, M.S., and Acworth, R.I. 2012. Experimental investigation of the thermal dispersivity term and its significance in the beat transport equation for flow in sediments. Water Resources Research. 48: W03511.

Stauffer, F., Bayer, P., Blum, P., Molina-Giraldo, N., and Kinzelbach, W. 2013. Thermal use of shallow groundwater. CRC Press.

Yuan, Z.G., Somerton, W.H., and Udell, K.S. 1991. Thermal dispersion in thick-walled tubes as a model of porous media. International journal of heat and mass transfer. 34(11): 2715-2726. 